\title{
interview
}

\section{Quantum tools for classical coherence}

\author{
Optical coherence theory has a long and proud tradition. Nature Photonics spoke to Ayman Abouraddy \\ and Kumel Kagalwala to learn about their recent work, which may reshape this established field.
}

Coherence theory has been around for a long time. Why change it?

Back in the mid-1800s, when James Clerk Maxwell was still a teenager, Sir George Gabriel Stokes published works on a number of aspects of light, including aberrations, the dynamical theory of diffraction, fluorescence, spectroscopy and polarization. The topic of optical coherence took off with the work of Emil Wolf in the mid-1900s. The ideas of coherence and polarization are in a marriage of convenience. Whenever you talk about coherence it's very important to consider polarization as well.

The community is fairly rooted in a few groups that hold steadfast opinions about the mathematics of optical coherence theory. However, in our opinion, the mathematical structure that people use today in this field is not well-suited to the task. Although this came about for historical reasons, which is normal, people have unfortunately been stuck in this mindset for the past 50 years. Emil Wolf did a great service to the community by introducing his correlation functions, but they are only convenient for a subset of problems. He himself realized that and published a paper in 2003 that reevaluated the situation, with polarization in the picture.

Spatial coherence is well-described by Emil Wolf's work. Polarization is also well-described by tools devised by Stokes. The moment you combine these degrees of freedom, the traditional measures we have for characterizing the different aspects of the field break down; these issues are not resolvable, in our opinion, without changes, because people are using the wrong, or at least not the most convenient, mathematical structure.

Tell us about your idea of bringing quantum mechanics tools over to classical optics.

What we did was identify the appropriate mathematical framework. We say 'identify' because it was already out there, in the nearby field of quantum optics. We believe that the mathematical framework used in quantum mechanics is the correct one to use for optical coherence,

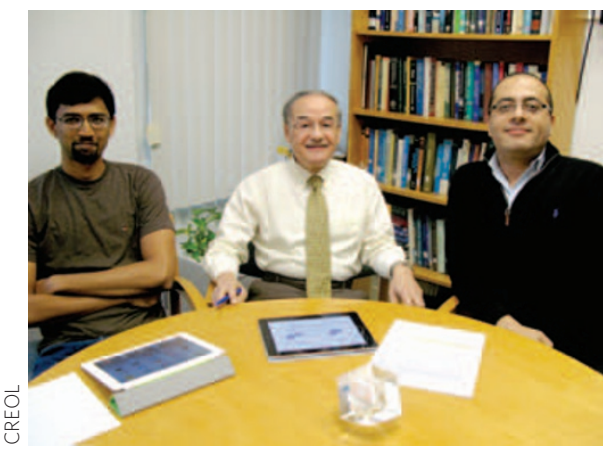

Left to right: Kumel Kagalwala, Bahaa Saleh, Ayman Abouraddy and Giovanni Di Giuseppe (not pictured) from CREOL, The College of Optics \& Photonics, University of Central Florida, USA. The researchers have shown in theory and experiments that the tools of quantum optics can be used for problems in classical optics.

at least when multiple degrees of freedom are involved.

We recently had a conversation with John Joannopoulos at the Massachusetts Institute of Technology in the USA, who has a condensed matter physics background and is well-known for his work on photonic crystals. In his book he showed that you can take Maxwell's equations and massage them into a form that is mathematically similar to Schrödinger's equation, which allowed him to apply the mathematics developed for electronic crystals to photonics. That helped optics quite a bit. However, that idea was not continued to its logical conclusion. Joannopoulos showed that looking at the spectral modes in an optical system can provide the equivalent of the one-particle Schrödinger equation. I posed the question to John: "What in optics is the equivalent to the two-particle Schrödinger equation?" Our work answers this question. The spatial and polarization degrees of freedom, which are at the centre of the debate in coherence theory today, correspond to two particles in the quantum context. All of the tools developed for two-particle states in quantum information science over the past few decades may now be of direct utility to optical coherence.
We have fired the first salvo in this friendly battle by showing you can calculate the correlation between the two 'particles' using tools from quantum information. Once you accept that Maxwell's equations for two degrees of freedom have the same structure as that of quantum theory for two particles, it's not difficult to calculate the correlation using Bell's inequality.

\section{What are the implications of your findings?}

We have shown that the correct way to identify a beam in which these two degrees of freedom might be mixed is to introduce interference that spreads across those two degrees of freedom. The outcomes of such measurements are scalar invariants that generalize the concept of visibility to multiple degrees of freedom. We have demonstrated something equivalent to Young's interference, but across two degrees of freedom. We have also shown that if you don't take this into account, your results might not be interpreted correctly in certain situations.

Our work is also relevant for coherent light - not only partially coherent light. One of the experiments in our manuscript was performed with a coherent source, but we found that it appeared partially coherent if we didn't take into account all entangled degrees of freedom together. We hope that our work allows people to understand some things they may have seen in their experiments.

\section{What will the community say when} they see this?

"Outrageous!" Nature Photonics will probably be inundated with comments. This work goes against the grain of what some people think. We hope that most scientists, after the initial shock, will sit down and give our work a careful read. We think it's exciting that the well-developed tools from quantum information are relevant to classical optics, where it is easier to perform experiments.

\section{INTERVIEW BY DAVID PILE} Ayman Abouraddy, Kumel Kagalwala and colleagues have an Article on using Bell's measure as a tool for classical optical coherence on page 72 of this issue 